\title{
Rythmes de croissance et de régénération des racines de plants et boutures de chênes pédonculés (Quercus pedunculata Ehrh.)
}

\author{
M. EL NOUR *,A. RIEDACKER ** \\ *Mâtre-Assisant a IUniversité de Khartoum (Soudan) \\ actuellement à la Station de Sylviculture et Production \\ **I.N.R.A., Station de Sylviculture et de Production \\ Centre de Recherches forestieres \\ Champenoux, f. 54280 Seichamps
}

\section{Résumé}

Nous avons observé la croissance aćrienne et souterraine de plants et boutures de jeunes chênes pédonculés (Quercus pedunculata Ehrh.) élevés en minirhizotrons :

- en conditions naturelles,

- en chambres climatisées 18 "C et jours longs,

- en serre non chauffée.

Nous avons également étudié la régénération de racines de jeunes chênes arrachés chaque semaine et repiqués en chambre climatisée à $20^{\circ} \mathrm{C}$ jours longs.

En conditions naturelles, bien que le nombre de pousses soit limité (3), les vagues de croissance aérienne restent bien synchronisées dans le temps.

Chez les plants, aussi bien que chez les boutures et quelles que soient les conditions, la croissance souterraine est déprimée pendant qu'ont lieu les vagues de croissance aérienne.

Du point de vue pratique, la plantation avec des racines blanches à l'automne permet d'avoir une bonne installation et reprise des plants; pour que les racines se régénèrent au mieux, il faudrait un chauffage particulier à $25{ }^{\circ} \mathrm{C}$ du système racinaire, cela après lia chute des feuilles, et avant le débourrement.

\section{Introduction}

Les chênes pédonculés et sessiles reprennent généralement assez bien en plantations, mais peuvent présenter des crises de transplantation importantes et prolongées (Rifoackir \& Polda, 1977). I1 en résulte des coûts de dégagement élevés. C'est pour réduire cette crise que nous nous intéressons à la croissance et à la régénération des racines de cette essence, après nous être intéressés antérieurement aux possibilités de modifier leur morphogénèse (RIEDACkER et al., 1982). 
La croissance aérienne du chêne est rythmique. même chez les jeunes individus (Lavarenne et al., 1971 ; Borchet, 1975 ; PAYAN, 1982). Les pivots des tout jeunes plants ont en revanche une croissance qui semble dépendre de la température. Elle est en fait indépendante de la croissance aérienne (Lavarenne, 1968). Hoffmann \& LYR (1974) avaient pourtant pris le chêne (Quercus typ) comme étant caractéristique des plants présentant une alternance de croissances souterraine et aérienne. Reich et al. (1980), en observant des arbres adultes dans la nature, ont constaté une réduction de la croissance souterraine pendant les phases de débourrement et d'étalement des feuilles. En outre, dans la nature, les très jeunes chênes ne présentent pas de vagues de croissance importantes avant le débourrement, alors que tel est le cas pour les chênes plus âgés.

La régénération des racincs de chênes Quercus rubra dépend étroitement de la température du sol (LARSON, 1970); $24^{\circ} \mathrm{C}$ semble être une température favorable en labsence de stress hydrique (LARSON, 1971).

\section{Matériels et méthodes}

Nous avons installé différents types de boutures enracinées en minirhizotrons (RIEDACKER, 1974), afin de pouvoir mesurer leur croissance aérienne et souterraine. simultanément, dans des conditions variées :

\subsection{Croissance de plants installés en conditions naturelles}

4 plants de un an ont été installés en avril 1982 dans la pépinière d'Amance, près de Nancy. Les minirhizotrons de $1,5 \mathrm{~m}$ de long étaient enterrés dans le sol, inclinés à $45^{\circ}$ et saturés en eau une fois par semaine. Les mesures ont été poursuivies jusqu’en décembre 1982 .

\subsection{Croissance de plants et boutures en chambre climatiséc $\left(18^{\circ} \mathrm{C}, 16 \mathrm{~h}\right.$ de jour à 2000 lux $)$}

La croissance de 5 plants et 5 boutures de un an a été suivie pendant 34 semaincs par des mesures hebdomadaires.

\subsection{Croissance des boutures en serre non chauffée, mais hors gel}

La croissance de 5 boutures a été mesurée chaque semaine. du 12 mai au 5 janvier.

\subsection{Régénération et croissance des racines de jeunes plants}

Chaque semaine, 6 plants de un an, élevés dans la pépinière de Champenoux, ont été repiqués en minirhizotrons et placés en chambre climatisée $\left(20^{\circ} \mathrm{C}-16 \mathrm{~h}\right.$ de jours - 5000 lux). L'opération implique des lésions importantes du système racinaire qui doit donc se régénérer. Certains lots de 5 plants ont en outre subi les traitements particuliers suivants : 

a) décapitation $1 \mathrm{~cm}$ au-dessus du collet;
b) décapitation $1 \mathrm{~cm}$ en dessous du collet:
c) partie aérienne maintenue à l'obscurité :
d) partie aérienne maintenue à 5 "C et partie souterraine maintenuc à 20 " $\mathrm{C}$.

\subsection{Plantation des chênes aver des pointes blanches à l'automne}

En septembre 1982, 5 plants de six mois semés en sachets en pépinière ont élé extraits sans léser les extrémités racinaires blanches et installés en minirhizotron dans la pépinière d'Amance. Les minirhizotrons étaient inclinés à $45^{\circ}$ el enterrés dans le sol. La croissance et la régénération ont été contrôlées chaque semaine. jusqu'en décembre 1982.

\subsection{Régénération des racines de grands plants de chênes (2 $\mathrm{m}$ de longueur)}

Issus de semis en 1974 et non repiqués depuis. ils ont été arrachés de la pépinière le 4 mars 1982. Leurs systèmes racinaires ont été coupés à $25 \mathrm{~cm}$ du collet, puis placés dans des sacs en polyéthylène contenant de la tourbe fertiliséc. La moitié inférieure des branches a été supprimée. Ils ont été ensuite placés pendant 40 jours par lot de 5 plants dans les 4 conditions suivantes :

- serre non chaufféc - racines chauffées à $25^{\circ} \mathrm{C}$;

- serre non chauffée - racines non chauffées :

- serre chaude (minimum $10^{\circ} \mathrm{C}$ la nuit), racines non chauffées:

- serre chaude (minimum $10^{\circ} \mathrm{C}$ la nuit), racines chaufées à $25^{\circ} \mathrm{C}$.

Nous avons mesuré la somme des allongements racinaires, le nombre de racines régénérées, le nombre de bourgeons débourrés et les sommes des allongements des pousses.

La croissance souterraine était relevée sur des feuilles de polyéthylène appliquées sur les faces transparentes de minirhizotrons, puis mesurées au curvimètre. Les résultats sont présentés sous forme de diagrammes indiquant simultanément les croissances aérienne et souterraine. Dans certains cas, nous avons lissé les données relatives aux systèmes racinaires par la formule suivante :

$$
\bar{x}_{1}=\frac{x_{i} 1+2 x_{i}+x_{i-1}}{4}
$$

et calculé la courbe des écarts $e_{i}=\bar{x}-x_{1}$ (l'intervalle $x_{i},-x_{i}$ vaut une semaine).

\section{Résultats}

\subsection{Plants en conditions naturelles (fig. 1 et 2)}

La croissance aérienne présente 3 vagues (fig. 1 A) bien synchronisées entre les différents plants. Lat croissance souterraine augmente avec la température du sol à 

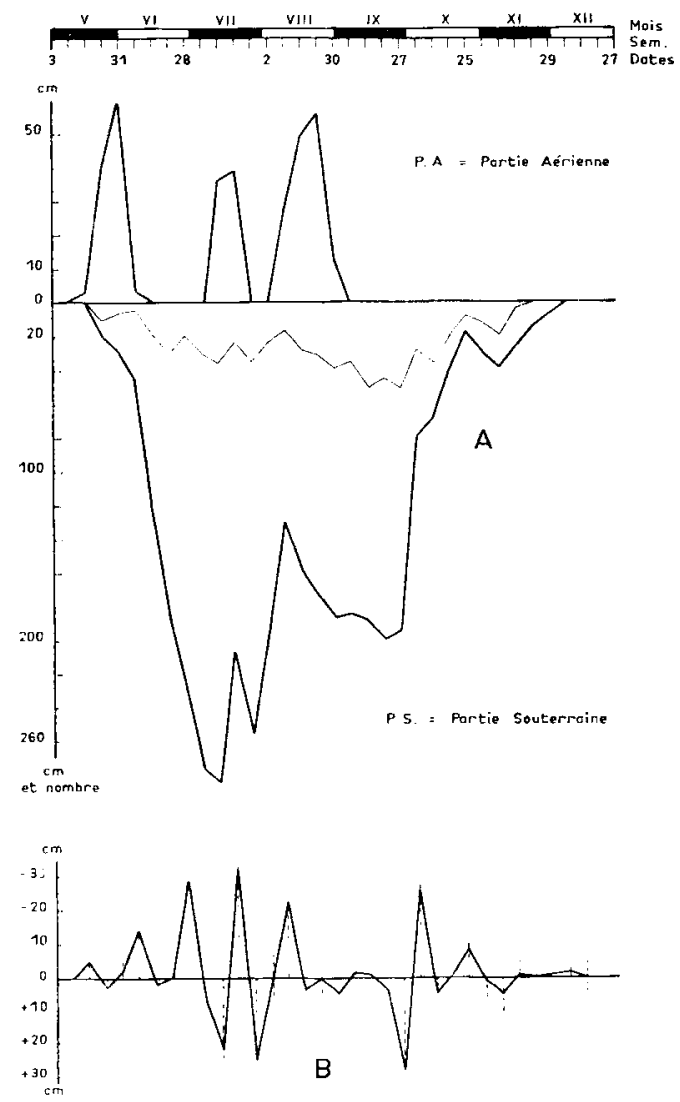

lisi. 1

Crovissance de jemes chênes en pépinières d'Amance.

Gronth of oak sectlings in Amance nursery.

Vagues apparaissant simultanément sur les 4 plants.

Partie aérienne (-) : somme des allongements aériens (pousses) par semaine.

Partic souterraine (-) : somme des allongements des racines par semaine:

(一) : nombre de nouvelles racines apparues au cours de la semaine.

A) Dialgramme moyen.

B) Ecarts entre l'allongement racinaire réel et la valeur moyenne lisséc

Simultaneons root elongation lagues on all the plants 14 plants). Acrial part (-) : shoot alongation per weeh.

Subterramean part (-) : root elongation per week:

(一) : number of newly formed roots per week.

1) Mean diagram of shoot and root growth.

B) Difference between original and smoothed values of root elongation. 


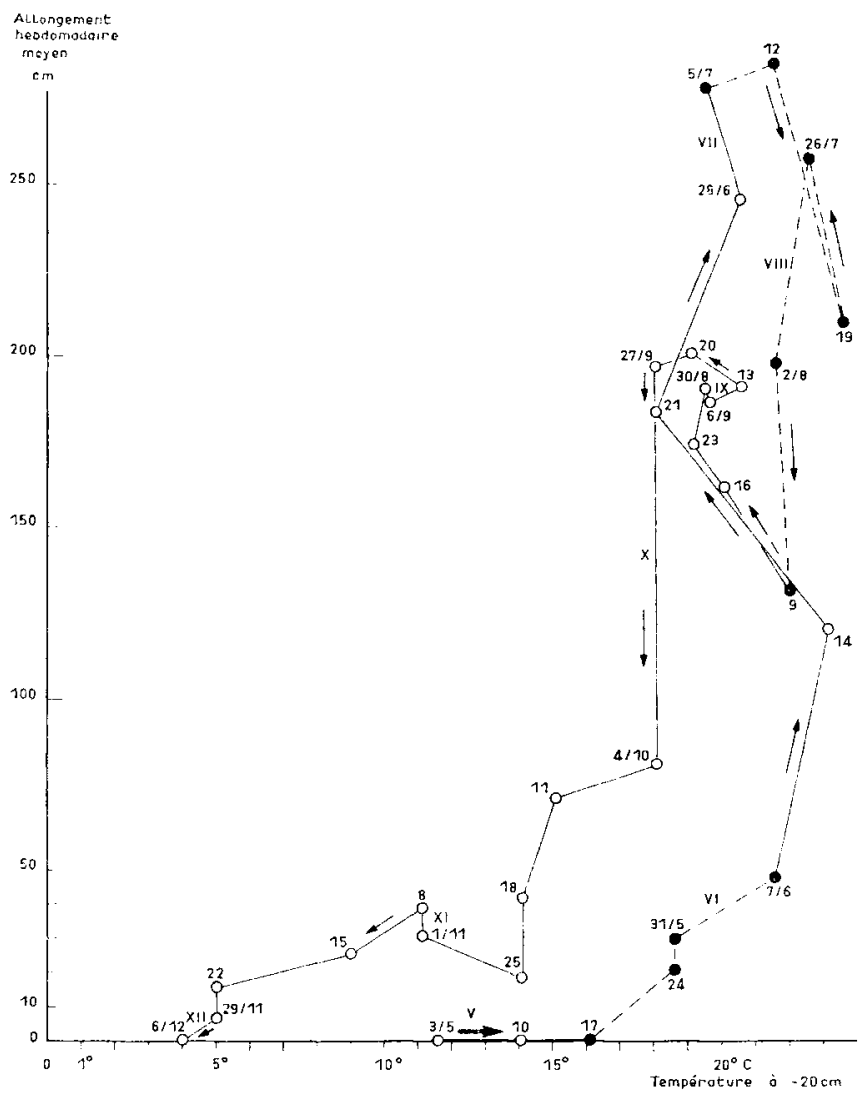

Fili. 2

Relation entre lallongement moyen des racines des chenes in situ et la température du sol à $20 \mathrm{~cm}$ de profondear.

Relatomship berween the mean root elongation of oak seedling in situ and soil temperanare at $20 \mathrm{~cm}$ of depth.

période diaccroissement soutertain simultanéc avec une période diaccroissement aćricn.

____ o- période d'accroissement souterain en l'absence d’accroissement aérien..

-...... : simultametots root growth with shoot growth.

- o- : root growth dering a complete absence of shoot elongation.

$20 \mathrm{~cm}$ de profondeur (fig. 2). Elle démarre à des températures élevées (18 "C) le 24 mai, postéricurement au débourrement qui a lieu le 17 mai. Elle est relalivement faible début juin pendant la première vague de croissance aérienne, puis augmente fortement vers la fin juin, pendant le premier arrêt de l'élongation aérienne. On remarque deux dépressions, l'une le 19 juillet, pendant la seconde vagute de croissance aérienne, et une autre plus importante le 9 août, quti coïncide avec le pie de la troisième vague de croissance aérienne. Deux autres dépressions (toujours de la 
croissance souterraine), situées l'une le 4 octobre et l'autre le 25 octobre peuvent être enregistrées. Ces dernières ne correspondent pas à des vagues de croissance aérienne. La figure 1 B montre que les vagues de croissance souterraine sont relativement bien synchronisées. Notons que la croissance souterraine à $1 \mathrm{~m}$ de profondeur se poursuit jusqu'à l'arrêt automnal le 6 décembre $\left(5^{\circ} \mathrm{C}\right)$. 11 semble cependant y avoir un ralentissement dès le 25 octobre. lorsque la température du sol descend en dessous de $15^{\circ} \mathrm{C}$.

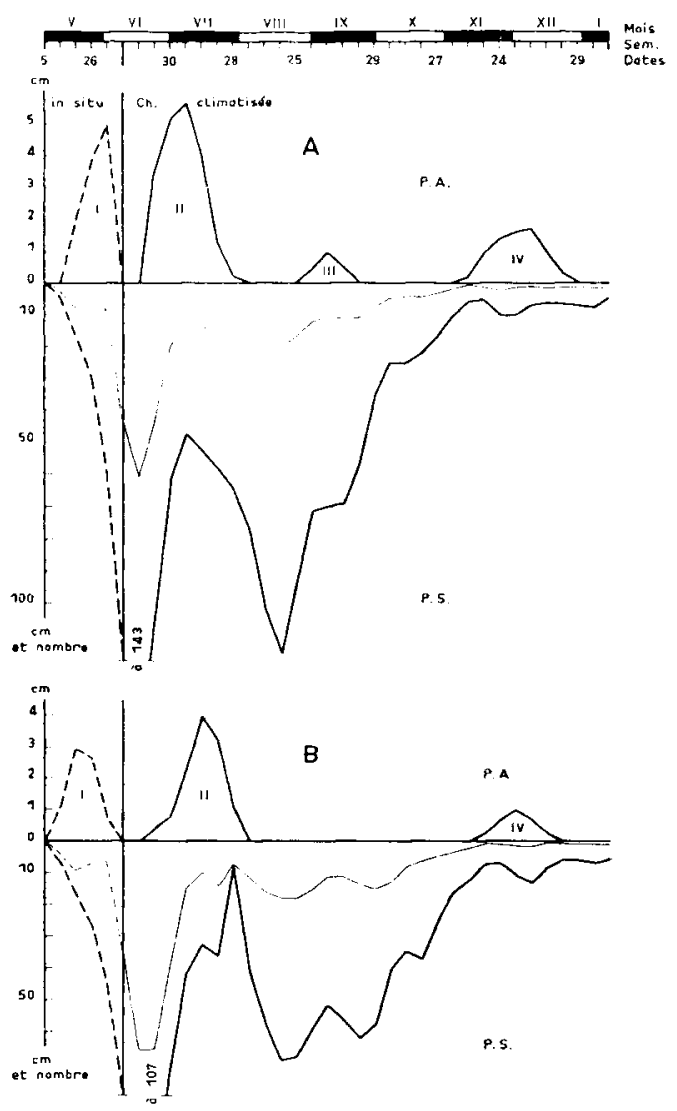

Fici. 3

Croissance de jeunes chênes en chambre climatisée $18^{\prime \prime} \mathrm{C}$ jours longs.

Growth of oak seedlings in a growth chamber at $18^{\circ} \mathrm{C}$, and long daration of light.

A) Diagramme moyen de tous les plants.

B) Diagramme individuel. (Noter la disparition de la vague III)

Partie aérienne (-) : somme des allongements aériens (pousses) par semaine.

Partie souterraine (-) : somme des allongements des racines par semaine:

(-) : nombre de nouvelles racines apparues au cours de la semaine.

A) Mean diagram of growth.

B) Individual diagram (note the disappearance of the vague $I I I)$.

Aerial part (-) : shoot elongation per week.

Subterranean part (-) : root elongation per week;

(-) : number of newly formed roots per week. 
3.2. Plants et boutures placés en chambre climatisée à $18^{~ " C}$ et jours longs

(fig. 3 et 4 )

La croissance aérienne se poursuit par vagues assez bien synchronisées. Mais, certaines vagues peuvent disparaître (fig. 3 B par exemple) pour tel ou tel plant.

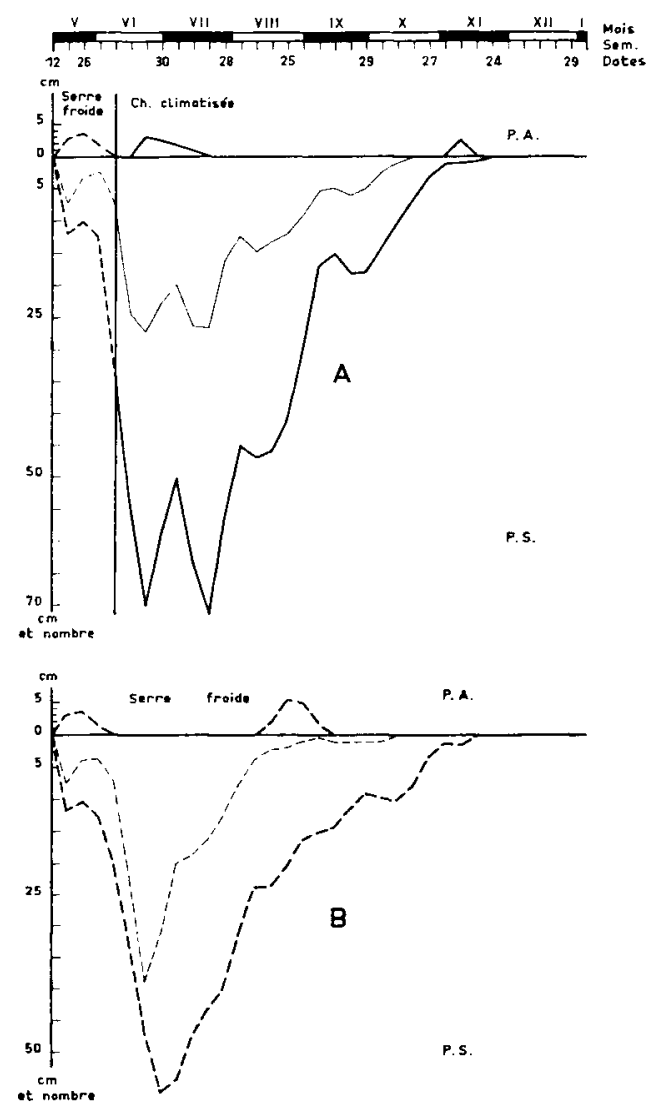

Fig. 4

A) Croissance des boutures en chambre climatiséc $18^{\circ} \mathrm{C}, 16 \mathrm{~h}$ d'éclairement (diagrammes moyens lissés).

B) Serre non chaufféc.

Partie aérienne (-) : somme des allongements aériens (pousses) par semaine.

Partie souterraine (-) : somme des allongements des racines par semaine;

(-) : nombre de nouvelles racines apparues au cours de la semaine.

Average smoothed growth diagram of pedoncule cuttings in :

A) Growth chamber at $18^{\circ} \mathrm{C}$ and $16 \mathrm{hl}$ light.

B) Frost-free glass house.

Aerial part (-) : shoot elongation per week.

Subterranean part (-) : root elongation per week:

(二) : number of newly formed roots per week. 
La croissance racinaire de juin précédant la première vague de croissance aérienne en chambre climatiséc est forte (fig. 3 . A et B). Tout se passe comme si nous assistions à la suite de croissance in situ indiquée en pointillés sur la figure 3.

Pendant la première vague de croissance aérienne en chambre climatisée, la dépression de la croissance souterraine est très visible. Mais, lorsqu'une vague de croissance aérienne disparaît (cas de la vague $11 \mathrm{I}$, fig. $3 \mathrm{~B}$ ), il n'y a pas de depression nette de la croissance racinaire. On notera que dans tous les cas, la croissance des racines est moins facilement lisible lorsque le système racinaire occupe tout le minirhizotron, et qu'il y a en même temps une diminution de la croissance racinaire. Les boutures (fig. $4 \mathrm{~A}$ ) se comportent en chambre climatisée sensiblement comme les plants.

\subsection{Bouture en serre non chauffée (hors gel) (fig. 4 B)}

On note comme précédemment une dépression de la croissance souterraine pendant la première vague de croissance aérienne. La croissance souterraine augmente, puis diminue, cela est explicable seulement en partie par les variations de température de la serre. En fait. aussi bien en chambre climatisé à température constante qu'en serre hors gel (fig. 4 , A et B), on assiste à une diminution progressive avec le temps des potentialités de croissance du système racinaire.

\subsection{Régénération des racines de jeunes chênes}

Entre le 21 octobre el le 14 avril, les plants repiqués en chambre climatisée ont un taux de survie de 100 p. 100 . Par contre. les plants transférés entre mai et août, c'est-à-dire après le débourrement, meurent tous en quelques jours. Les feuilles des plants transférés le 18 septembre se dessèchent. puis il y a un nouvealu débourrement le 23 octobre. Mais, seulement deux plants sur six survivent.

Quant à la régénération des racines, elle peut précéder légèrement la croissance aérienne (fig. $5 \mathrm{a}, \mathrm{A}$ ) ou démarrer simultanément (fig. $5 \mathrm{a}, \mathrm{B}$ ). Mais, cette première phase de croissance racinaire peut aussi disparaitre complètement (fig. 5 a, C). Dans tous les cas. elle est suivie d'une dépression correspondant au pic de la vague de croissance aćrienne (fig. 5 a), ou décalée vers la fin de celle-ci (lig. 5 B). La seconde phase de croissance racinaire est en général plus importante que la première.

Le sectionnement de la partie aérienne att-dessus du collet se traduit par une croissance aérienne el souterraine normale (fig. 5, D). La suppression complète de la partie aćrienne par une section en dessous du collet entraîne en revanche un arrêt total de la croissance racinaire chez beaucoup de plants et/ou une croissance très faible chez les autres, $3 \mathrm{~cm}$ en moyenne (fig. $5 \mathrm{~b}, \mathrm{D}^{\prime}$ ).

Lorsque la partie aérienne est maintenue intacte, mais au froid (fig. $5 \mathrm{~b}$. T), lc débourrement est retardé et la croissance souterraine est plus faible, mais se prolonge pendant plusieurs semaines. Après transfert le 30 avril à $20^{\circ} / 20^{\circ} \mathrm{C}$, on assiste à une reprise de lactivité aérienne suivie par une très forte croissance racinaire. Enfin, lorsque le débourrement a lieu à l'obscurité (fig. 5 b, O), la croissance souterraine cesse après la première phase qui esı normale. La partie aérienne en revanche présente une seconde vague, mais s'étiole faiblement. 

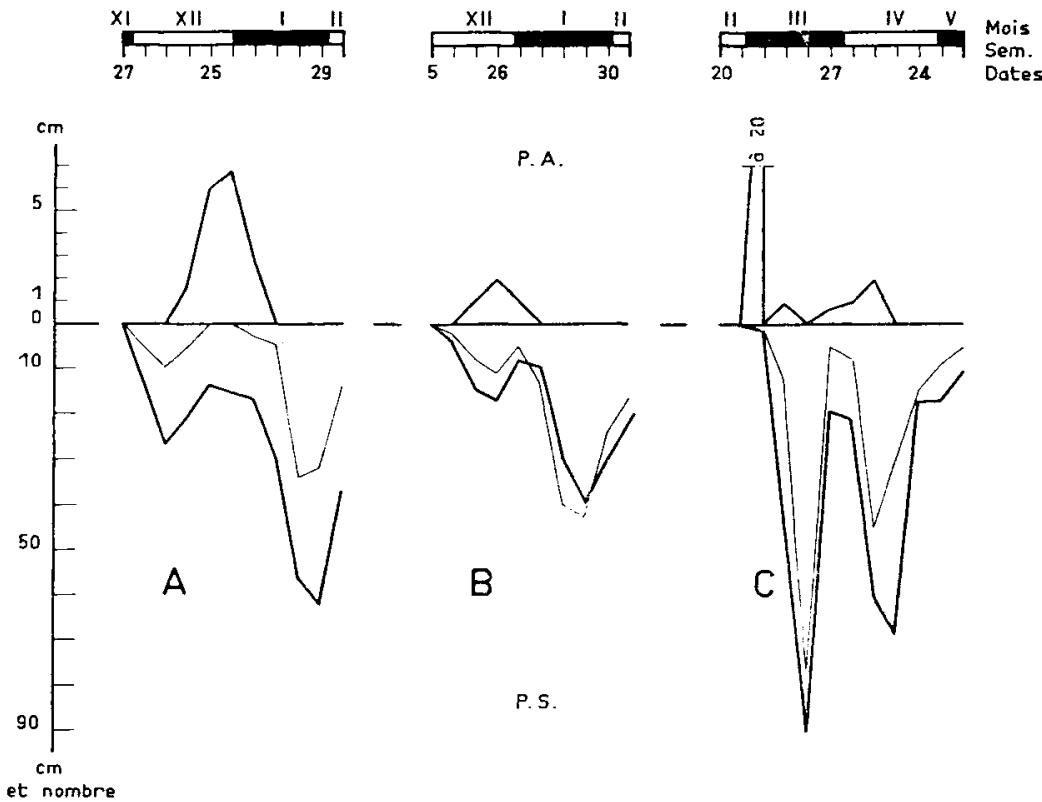

FIG. 5 a

Régénération de racines de jeunes chênes en conditions constantes $\left(20^{\circ} \mathrm{C}, 16 \mathrm{~h}\right.$ d'éclairement 4000 lax).

Root regeneration of oak seedlings under controlled conditions $\left(20^{\circ} \mathrm{C}\right.$ and $16 \mathrm{~h}-$ light 4000 lux $)$.

A) La première vague de croissance précède nettement la vague de croissance aérienne.

B) La première vague de croissance aérienne et souterraine sont presque concomittantes.

C) La croissance souterraine ne débute qu’après la première vague de croissance aérienne.

Partic aérienne (-) : somme des allongements aériens (pousses) par semaine.

Partic souterraine (-) : somme des allongements des racines par semaine:

(一) : nombre de nouvelles racines apparues au cours de la semaine.

A) The first vague of root growth precede that of shoot growth.

B) Root and shoot vagues are simultaneous.

C) No root growth observed before the end of the first vague of shoot growth.

Aerial part (-) : shoot elongation per week.

Subterranean part (-) : root elongation per week;

(一) : number of newly formed roots per week. 


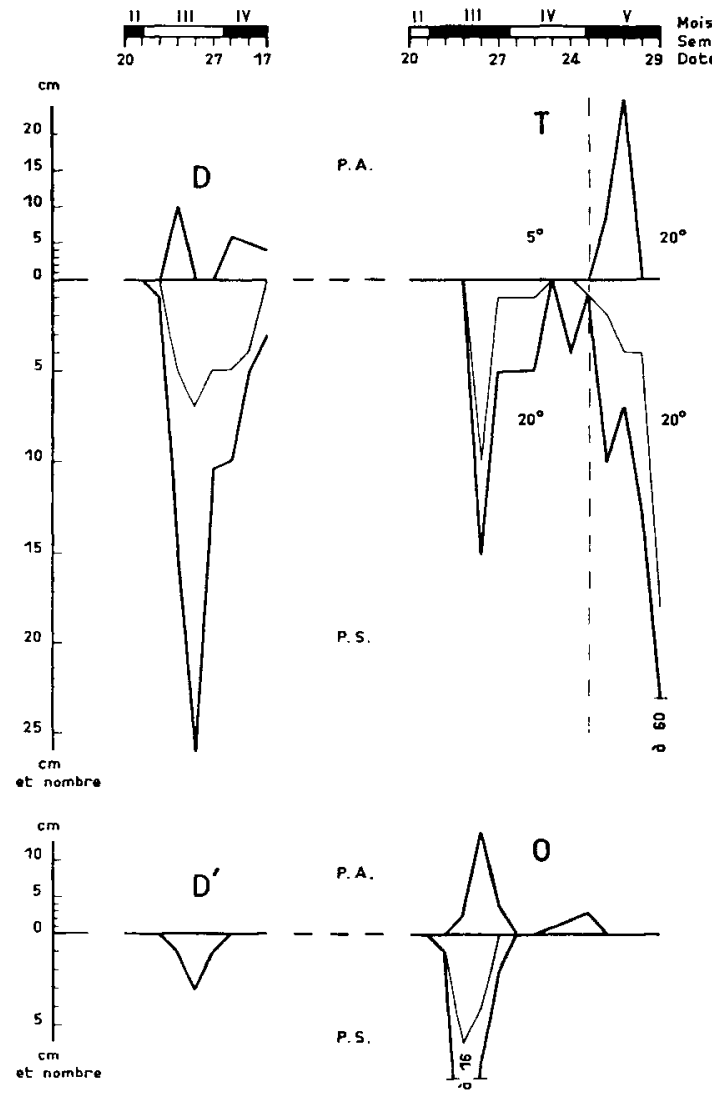

Fig. $5 \mathrm{~b}$

D) Plants ayant été décapités à $1 \mathrm{~cm}$ au-dessus du collet.

D') Plants ayant été décapités à $1 \mathrm{~cm}$ en dessous du collet.

T) Plants placés à 5 " $\mathrm{C}$ (partie aérienne) et $20^{\circ} \mathrm{C}$ (partie soutcrraine) jusqu'au 30 avril, puis transférés à $20^{\circ} \mathrm{C}$.

O) Plants placés à l'obscurité.

Partie aérienne (-) : somme des allongements aériens (pousses) par scmaine.

Partie souterraine (-) : somme des allongements des racines par semaine;

(二) : nombre de nouvelles racines apparues au cours de la semaine.

Root regeneration of oak plants when :

D) Tops were cut off $1 \mathrm{~cm}$ above the root collar.

D') Tops were cut off $1 \mathrm{~cm}$ below the root collar.

T) Plants were placed at $5{ }^{\circ} \mathrm{C}$ (aerial part) and $20^{\circ} \mathrm{C}$ (subterranean part) up to 30 April and transferred there after to $20^{\prime \prime} C$ for both parts.

O) Plants were grown in complete darkness.

Aerial part (-) : shoot elongation per week.

Subterranean part (-) : weekly elongation of roots;

(-) : number of newly formed roots per neek. 
3.5. Plantation de jeunes chênes avec des pointes racinaires blanches à l'automne (fig. 6)

Les pointes racinaires blanches continuent à s'allonger et à donner de nombreuses nouvelles racines jusqu'à la fin de novembre. La partie aérienne reste en repos apparent.
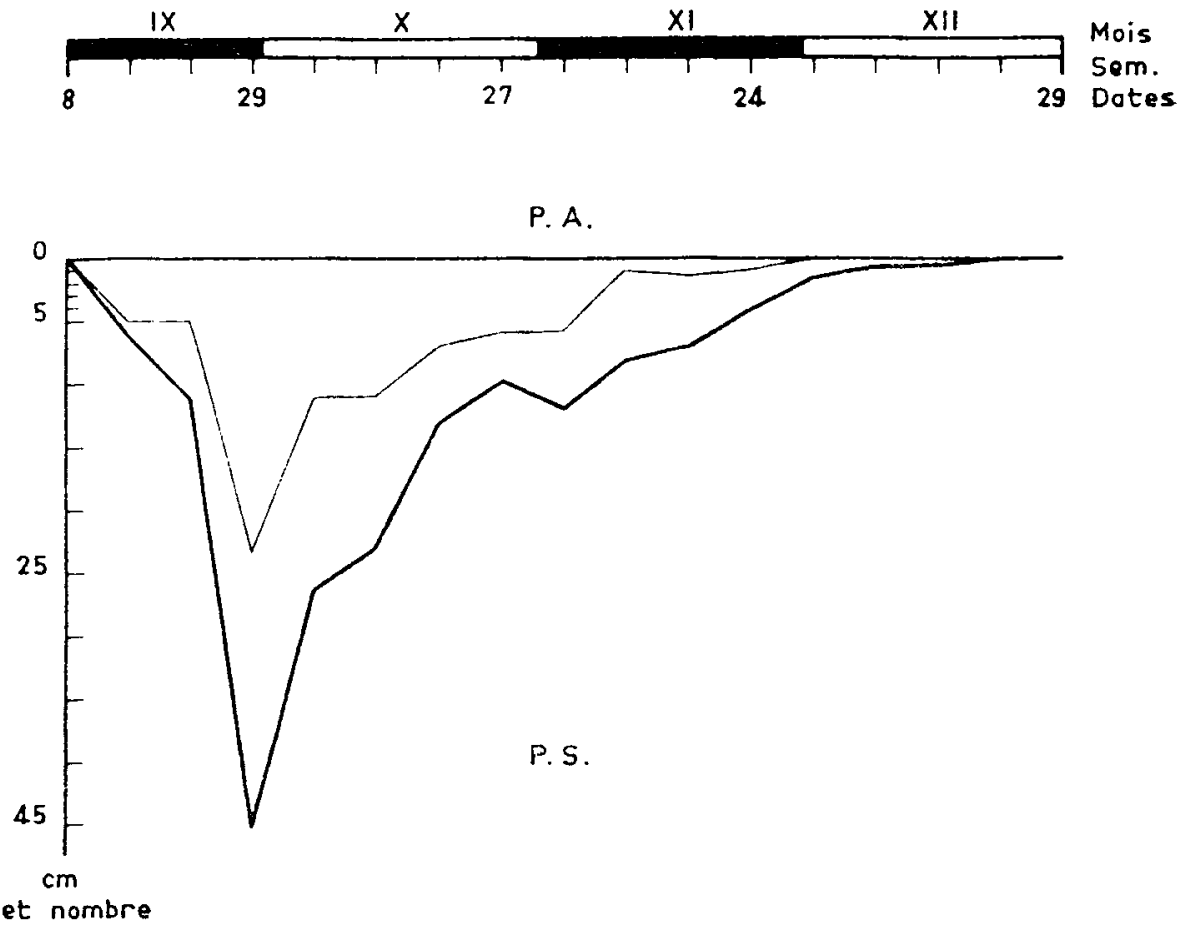

FIG. 6

Diagranme moyen de croissance de chênes de 6 mois en pépinière d'Amance repiqués le 2 septembre.

Mean growth diagram of 6 months old oak seedlings, transplanted in Amance nursery in September 2nd.

Partic aériennc : pas de croissance aérienne en automne.

Partie souterraine (-) : somme des allongements des racines pat semaine;

$(-)$ : nombre de nouvelles racines apparues au cours de la semaine.

Aerial part : no aerial growth in autumn.

Subterranean part (-) : weekly root elongation;

(-) : number of newly formed roots per week. 

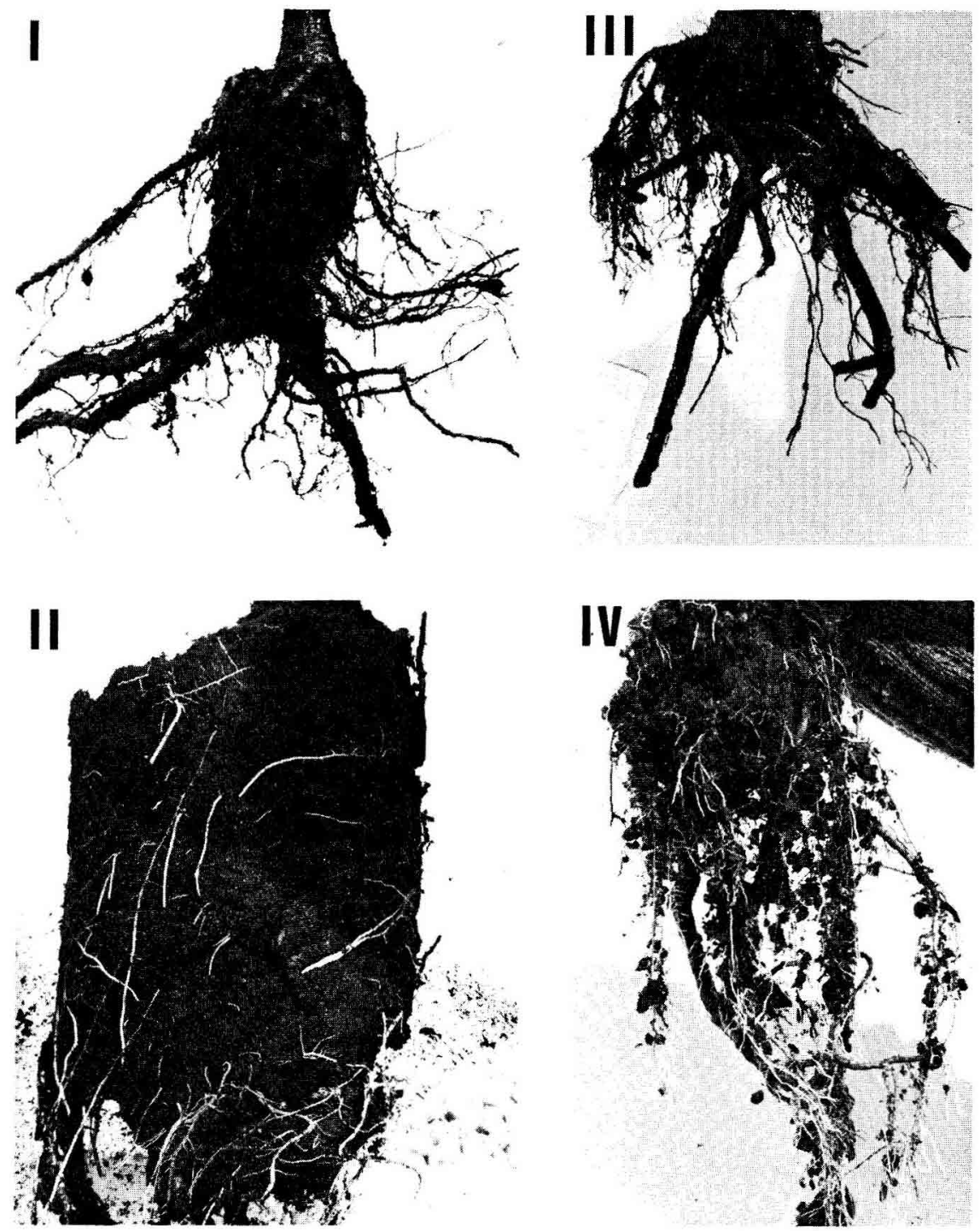

FIG. 7

Régénération de racines de chênes de 7 ans. Root regeneration of 7 years old pedunculate plants.

I : Sans chauffage de racines en serre non chauffée.

Without a localized heating of roots in a frost-free glasshouse.

II : Racincs à $25^{\circ} \mathrm{C}$ en serre non chauffée.

With a localized heating of roots at $25^{\circ} \mathrm{C}$ in frost-free glasshouse.

III : Sans chauffage de racines en serre chaude.

Without a localized heating of roots in a heated glasshouse.

IV : Racines à $25^{\circ} \mathrm{C}$ en serre chaude.

With a localized heating of roots at $25^{\circ} \mathrm{C}$ in heated glasshouse. 


\subsection{Régénération des racines des grands chènes \\ ( $2 \mathrm{~m}$ de longueur après 7 ans)}

En l'absence de chauffage des racines en serre non chauffée, 3 plants sur 5 ne régénèrent pas de racines et ne débourrent pas. Deux planis régénèrent 4 à 6 racines de longueur moyenne de $1 \mathrm{~cm}$ (fig. 7, 1), el débourrent après 30 jours. En serre chaude, mais sans chauffage particulier des racines, 2 plants sur 5 ne régénèrent pas de racines. Trois sur cinq régénèrent quelques racines de longueur moyenne $5,2 \mathrm{~cm}$ (fig. 7, III).

Lorsqu'on élève la température des racines à $25^{\circ} \mathrm{C}$, aussi bien en serre chaude qu'en serre maintenue hors gel, tous les plants régénèrent beaucoup de racines. Dans ce dernier cas, elles apparaissent à la surface de la motte de tourbe le 22 mars, soit 18 jours après le début de chauffage des racines (fig. 7,111 ). Les plants ont en moyenne 48 racines nouvelles par plant (36 a 63), d'une longueur moyenne de $13 \mathrm{~cm}$. Tous les plants ont débourré le 30 mars, c'est-à-dire une semaine après la régénération de racines observée le 22 mars. En serre chaude, avec élévation de la température de racines à $25^{\circ} \mathrm{C}$, il apparaît 24 à 80 racines par plant de longueur moyenne de $9,9 \mathrm{~cm}$ (fig. 7, IV). Tous les plants ont débourré le 16 mars, c'est-à-dire plus tôt, non seulement que dans la serre non chaufiée, mais aussi que dans la serre chaude en l'absence de chauffage particulier des racines. Le tableau 1 résume les caractéristiques de ces plants.

\section{TAlsLEAU 1}

Inflaence de la température souterraine et aérienne sur le développement dés joumes chênes (après 40 jours de chauffage de racines).

Influence of soil and aerial temperatures on the development of aok seedlings (40 days after a localized heating of roots).

\begin{tabular}{|c|c|c|c|c|c|c|}
\hline & \multicolumn{3}{|c|}{ Partie aérienne } & \multicolumn{3}{|c|}{ Partie souterraine } \\
\hline & $\begin{array}{l}\text { Nombre de } \\
\text { bourgecons } \\
\text { débourrés } \\
\text { par plant }\end{array}$ & $\begin{array}{c}\text { La pousse } \\
\text { la plus } \\
\text { longue } \\
\text { (em) } \\
\text { par plant }\end{array}$ & $\begin{array}{l}\text { Ia somme } \\
\text { des allon- } \\
\text { genents de } \\
\text { pousse par } \\
\text { plant }(\mathrm{cm})\end{array}$ & $\begin{array}{l}\text { Nombre de } \\
\text { nouvelles } \\
\text { racines } \\
\text { moyen } \\
\text { par plant }\end{array}$ & $\begin{array}{c}\text { Somme des } \\
\text { allonge- } \\
\text { ments par } \\
\text { piant (cm) }\end{array}$ & $\begin{array}{l}\text { Longueur } \\
\text { moyenne } \\
\text { par racine } \\
(\mathrm{cm})\end{array}$ \\
\hline $\begin{array}{l}\text { Elévation de tem- } \\
\text { pérature des raci- } \\
\text { nes à } 25^{\circ} \mathrm{C} \text { en } \\
\text { serre chaude ... }\end{array}$ & 79 & 31 & 363 & 58 & 577,6 & 9,9 \\
\hline 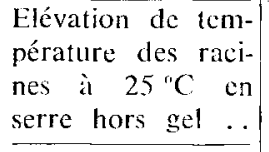 & 133 & 17 & 316 & 48 & 647,6 & 13,5 \\
\hline $\begin{array}{l}\text { En serre chaude } \\
\text { sans chauffage par- } \\
\text { ticulier des racines }\end{array}$ & 10 & 16 & 36 & 11 & 57,4 & 5,2 \\
\hline $\begin{array}{l}\text { En serre hors gel } \\
\text { et sans chauffage } \\
\text { particulier des ra- } \\
\text { cines ............ }\end{array}$ & 10 & 16 & 21 & 2 & 2,2 & $1,1$. \\
\hline
\end{tabular}




\section{Discussion et conclusion}

En conditions naturelles, la croissance des pousses est bicn synchroniséc, mais en nombre de vagues limité. En conditions contrólées, $25^{\circ} \mathrm{C}$, jour long, cette croissance rythmique peut se poursuive, mais les individus se déphasent (PAYAN, 1982). Dans nos conditions expérimentales, il arrive parfois, dans tel ou tel cas particulier, que certaines vagues de croissance á́rienne disparaissent.

Pendant les vagues de croissance aérienne, la croissance souteraine est dépriméc plus ou moins fortement. Cêla pourrait être dû :

- soit à une modification d'équilibres hormonaux:

- soit à des phénomènes de compétition de nature trophique.

Le fait que la croissance des racines s'annule à la suite d'une section en dessous du collet, et non à la suite d'une décapitation immédiatement au-dessus du collet (en l'absence de nouvelles feuilles) montre que des phénomènes hormonaux interviennent effectivement. Et, si au printemps, la croissance des racines de jeunes chênes in situ (en pépinière) démarre après le débourrement «aérien», il n’en va pas de même lorsqu'on chauffe les racines ou lorsquion est en présence de chênes adultes in situ (en pépinière). Dans e dernier cas, il est possible que les nombreux bourgeons des grands plants exercent un effet plus marqué sur le démarrage de la croissance racinaire. Mais, il est possible aussi que le stock de glucides utilisables pour la croissance des racines soit plus important au printemps chez les arbres de grande taille que chez les semis.

Une faible régénération et croissance des racines, lorsque la partic aéricnne non débourrée est maintenue à 5 " $\mathrm{C}$, une absence complète de seconde phase de croissance racinaire lorsque le débourremnt a lieu à l'obscurité, une croissance non rythmique chez les jeunes plants (LAVARENNE, 1968 ; RICHARDSON, 1956) qui disposent encore de réserves cotylédonaires sont autant d'arguments en faveur de l'hypothèse « compétition trophique».

Rappelons aussi qu'en cas d'ombrage de jeunes chênes âgés de plus d'un an, la croissance aérienne s'annule complètement à la fin des deux premières vagues de croissance aérienne (HOFfmANn, 1967). En pleine lumière, les mêmes plants présentent trois vagues successives de croissance aérienne.

Notons également que la croissance souterraine continue à présenter parfois une ou deux phases de dépression, même en l'absence de croissance aérienne. Il est possible alors, bien que les conditions externes (température, lumière) ne soient plus tout à fait suffisantes pour l'expression de nouvelles vagues de croissance aérienne, que tout se passe encore au sein de la plante comme si les vagues devaient avoir licu normalement. Nous sommes alors dans une situation limite conduisant en principe à l'entréc en dormance des bourgeons.

Sur le plan pratique, il apparaît :

- que la plantation à l'automne de plants de chênes en mottes avec des pointes de racines blanches permet d'obtenir une bonne installation de plants avant la période de débourrement aérien de l'année suivante; 
- que pour obtenir une bonne régénération des racines de jeunes chênes, après la chute des feuilles et avant le débourrement, il est favorable d'élever la température des racines : $25^{\circ} \mathrm{C}$ semble une température tout à fait convenable. On peut conserver simultanément la partie aérienne au froid pour éviter de hâter le débourrement. Cette technique devrait permettre de transplanter des grands plants avec plus de chances de succès.

\section{Summary \\ Growth and regeneration rhythms of roots of seedlings and cuttings of pedunculate oak (Quercus pedunculata Ehrh.)}

The relationship between shoot and root growth was evaluated in Quercus pedunculata seedlings and cuttings under both natural as well as controlled conditions. Root regeneration was also studied in a growth chamber at $20^{\circ} \mathrm{C}$ and $16 \mathrm{~h}$ of light.

Under natural conditions the seedlings underwent three synchronous flushes of shoot growth. In the field greenhouse and in growth chamber root growth (elongation and regeneration) was reduced during shoot flushes.

From practical point of view fall plantation with roots having white extremities seems to allow best establishement of the seedlings in the field. However to obtain good regeneration of root just before plantation, localized heating of roots at $25^{\circ} \mathrm{C}$ is needed which can be done after the fall of leaves but before the bud break.

Accepté le 5 janvier 1984.

Reçu le 6 juin 1983.

\section{Références bibliographiques}

Borcher' R., 1975. Endogenous shoot growth rythms and indeterminate shoot growth in Oak. Physiol. Plant., 35, 152-157.

Hofrmann G., 1967. Wurzel und Sprosswachstumperiodik der Jungpflanzen von Quercus robur L. im Freiland und urter Schallenbelastung. Arch. Forstwes., 16, 745-749.

HofFman G., LYR H., 1974. Wachstumsschemata zur Darstellung des jahreszeitlichen Verlaufes verschiedener Zuwachsgrössen von Gehölzen. Symposium Postdam. 1971. Ecology and physiology of root growth. Akademie Verlag, Berlin, 275-282.

LARSON M.M., 1970. Root regeneration and early growth of red oak seedling; influence of soil temperature. For. Sci., 16, 442-446.

LaRson M.M., 1971. Moisture stress on oak seedlings. Reprint from Ohio Report, 56 (3) : 46-47, May-June 1971. Ohio Agricultural Research and development center. Wooster Ohio.

Lavarenne S., 1968. Croissance comparée des tiges et des racines des jeunes chênes cultivés en conditions contrôlées. C.R. Acad. Sci., Paris, T. 226, 778-780.

Lavarenne S., Champagnat P., Barnola P., 1971. Croissance rythmique de quelques vćgétaux ligneux des régions tempérées cultivés en chambre climatisće à température élevée et constante dans différentes photopériodes. Bull. Soc. Bot. Fr., 118 (3-4), $131-162$.

PAYAN E., 1982. Contribution à l'étude de la croissance rythmique chez de jetnes chênes pédonculés Quercus pedunculata Ehrh. Thèse Doct. spécialisé Univ. Clermont II, 102 p. 
Reich P.B., Teskey R.O., Johnson P.S., Hinckley J.M., 1980. Periodic root and shoot growth in Oak. For. Sci., vol. 26, n" 4, 1980, pp. 590-598.

Richakdson S.D., 1956. On the role of the Acron in root growth of American Oak seedlings. Meded. Landbouwhogesch. Wageningen, 56 (12), 1-18.

Riedacker A., 1974. Un nouvel outil pour l'étude des racines et de la rhizosphère : Ie minirhizotron. Ann. Sci. For., 31 (2), 129-134.

Riedacker A., Poda U., 1977. Les systemes racinaires de jeunes plants de hêtre et de chêne. Modification de leur morphogénèse par décapitation d'extrémités de racines et conséquences pratiques. Ann. Sci. For., 34 (2), 111-135.

Riedacker A., Dexheimer J., Tavakol R., Alaoui H., 1982. Modifications expérimentales de la morphogénèse et de géotropismes dans le système racinairc de jeunes chênes. Can. J. Bot., 60, 765-778. 